\title{
Anhang 1: Interviewleitfaden
}

- Aufzeichnung als Erinnerungsstütze an das Gespräch

- Anonymität

- Wie würdest du deine eigene Geschlechtsidentität bezeichnen und wie ist sie dir bewusst geworden?

- Wann wurde in dem Kontext das Thema der Namenfindung für dich wichtig? Welche Gedanken hast du dir da gemacht?

- Wie bist du dann letztendlich zu dem neuen Namen gekommen? (Hast du andere um Rat gefragt? Oder Bücher/das Internet durchsucht?)

- Was war dir wichtig am neuen Namen? (Der Klang? Nachname? Bezug zum alten Namen? Wunsch der Eltern?...)

- Offizielle Namensänderung? Nach TSG oder anders?

- Wie ist das Verhältnis zum alten Vornamen? Wenn er noch besteht: Wie fühlt er sich an? Oder: Wie hast du dich mit ihm gefühlt, hat sich das durch die Beschäftigung mit dem Thema trans verändert?

- Publikation des Namens? Zu welchem Zeitpunkt deines Coming-Outs hast du den Namen anderen mitgeteilt? Bewusste Entscheidung?

- Vorher Sorgen diesbezüglich? Gefühl dabei?

- Wem, wann, wo zuerst?

- Reaktion Umfeld? Wer weiß alles vom Namen/Benutzt ihn?

- Gibt es Durcheinander bei verschiedenen Namen? Reagierst du unterschiedlich auf die verschiedenen Namen? Wer verwendet welchen und warum?

- Dauer der Gewöhnung an den neuen Namen für dich und andere? Reaktionen?

- Personalpronomen? Welche/keine? Wie setzt du das um? Unstimmigkeiten zwischen Namen- und Pronomengebrauch?

- Artikel beim Namen? Der/die (Name)?

- Zufriedenheit mit dem Namen? Beim ersten Namen geblieben oder Wechsel? Falls ja, Gründe für einen Wechsel? Bist mit dem neuen Namen zufriedener?

- Spitznamen? Schon vorm Coming-Out? Wie ist er entstanden? Verwendung?

- Gibt es Menschen, die sich weigern, Ihren neuen Namen zu verwenden? Haben Sie spezielle „Namen-Abkommen“ mit einzelnen Menschen? 
